ARTICLE

DOI: $10.1038 / s 41467-018-03632-y$

\title{
Structural characterization of a highly-potent V3- glycan broadly neutralizing antibody bound to natively-glycosylated HIV-1 envelope
}

Christopher O. Barnes 1, Harry B. Gristick 1, Natalia T. Freund 2,5, Amelia Escolano 2, Artem Y. Lyubimov ${ }^{3}$, Harald Hartweger ${ }^{2}$, Anthony P. West Jr. ${ }^{1}$, Aina E. Cohen ${ }^{3}$, Michel C. Nussenzweig ${ }^{2,4}$ \& Pamela J. Bjorkman ${ }^{1}$

Broadly neutralizing antibodies (bNAbs) isolated from HIV-1-infected individuals inform HIV-1 vaccine design efforts. Developing bNAbs with increased efficacy requires understanding how antibodies interact with the native oligomannose and complex-type $\mathrm{N}$-glycan shield that hides most protein epitopes on HIV-1 envelope (Env). Here we present crystal structures, including a 3.8- $\AA$ X-ray free electron laser dataset, of natively glycosylated Env trimers complexed with BG18, the most potent V3/N332 gp120 glycan-targeting bNAb reported to date. Our structures show conserved contacts mediated by common D gene-encoded residues with the N332 gp120 glycan and the gp120 GDIR peptide motif, but a distinct Env-binding orientation relative to PGT121/10-1074 bNAbs. BG18's binding orientation provides additional contacts with $\mathrm{N} 392_{\text {gp120 }}$ and N386gp120 glycans near the V3-loop base and engages protein components of the V1-loop. The BG18-nativelyglycosylated Env structures facilitate understanding of bNAb-glycan interactions critical for using V3/N332 gp120 bNAbs therapeutically and targeting their epitope for immunogen design.

\footnotetext{
${ }^{1}$ Division of Biology and Biological Engineering, California Institute of Technology, Pasadena, CA 91125, USA. ${ }^{2}$ Laboratory of Molecular Immunology, The Rockefeller University, New York, NY 10065, USA. ${ }^{3}$ Stanford Synchrotron Radiation Lightsource, 2575 Sand Hill Road, Menlo Park, CA 94025, USA. ${ }^{4}$ Howard Hughes Medical Institute, The Rockefeller University, New York, NY 10065, USA. ${ }^{5}$ Present address: Department of Clinical Immunology and Microbiology, Sackler Faculty of Medicine, Tel Aviv University, Ramat Aviv, Tel Aviv 6997801, Israel. Correspondence and requests for materials should be addressed to P.J.B. (email: bjorkman@caltech.edu)
} 
$\mathrm{n}$ the ongoing fight against the HIV-1 pandemic, the discovery and characterization of broadly neutralizing antibodies (bNAbs) against HIV-1 envelope (Env) fuel new efforts in treatment and management of infection. Next-generation bNAbs protected against and reduced viral loads in humanized mouse $\mathrm{e}^{1,2}$ and non-human primate ${ }^{3,4}$ models of infection and exhibited anti-viral activity in human trials ${ }^{5-9}$. Therefore, a vaccine that elicits such antibodies is likely to be efficacious. Despite their promise, unusual properties of HIV-1 bNAbs-such as a high degree of somatic hypermutations ${ }^{10}$, long heavy chain complementarity determining region 3 (CDRH3) loops ${ }^{11}$, and/or short light chain complementarity determining region 3 (CDRL3) loops ${ }^{12}$-have made it difficult to elicit bNAbs by immunization. In addition, innate features of the viral Env spike that interfere with broad-based immunity include the diversity of Env sequences that arise by mutation ${ }^{13}$, a low Env density on the virion surface that interferes with bivalent antibody binding ${ }^{14,15}$, and host glycans that shield most of the Env surface ${ }^{16}$.

The glycan shield comprises $\sim 50 \%$ of the mass of HIV- 1 Env, a (gp120-gp41) $)_{3}$ trimer, and consists of $\mathrm{N}$-glycans attached to an average of $30 \pm 3$ potential $N$-linked glycosylation sites (PNGSs) per gp120-gp41 protomer ${ }^{17}$. The carbohydrates decorating the surface of Env reduce access to protein epitopes and are generally non-immunogenic because they are assembled by host cell machinery ${ }^{16}$. Although under-processed oligomannose $N$-glycans cover parts of HIV-1 Env such as the N332 $2_{\text {gp120 }}$ glycan region of gp120, processed complex-type $N$-glycans predominate in other regions of Env ${ }^{18}$ and protect the CD4 binding site (CD4bs) and the variable loop 3 (V3-loop) of gp $120^{19}$. The production of soluble native-like Env trimers (SOSIPs) ${ }^{20}$ has enabled structure determinations of glycosylated Env-bNAb complexes and a better understanding of bNAb epitopes ${ }^{11,21-24}$. However, structural information pertaining to $\mathrm{bNAb}$ recognition of highly glycosylated HIV-1 Env trimers in the context of native glycan shields has been difficult to obtain due to chemical and conformational heterogeneity of $\mathrm{N}$-glycans that usually precludes crystallization required for an X-ray structure determination. Thus with one exception $^{25}$, all monomeric and trimeric Env crystal structures were solved using glycoproteins produced with exclusively high mannose-only forms of $\mathrm{N}$-glycans, which were usually enzymatically trimmed after being complexed with antibody Fabs ${ }^{22,26-37}$. A single-particle cryo-electron microscopy (cryo-EM) structure of a natively glycosylated HIV-1 Env showed complex-type $\mathrm{N}$-glycans at the gp120-gp41 interface, but many of the remaining glycans were not ordered in the EM map ${ }^{38,39}$.

The V3/N332 $2_{\text {gp } 20}$ class of HIV-1 bNAbs, exemplified by the PGT121/10-1074 family ${ }^{40,41}$, evolved to interact with both glycan and protein components on HIV-1 Env to effect the neutralization of a majority of HIV-1 strains ${ }^{31,42-44}$. These bNAbs possess long CDRH3s that interact with the $\mathrm{N} 332_{\text {gp } 120}$ glycan and penetrate the glycan shield to contact the conserved ${ }^{324} \mathrm{GD} /$ $\mathrm{NIR}^{327}$ peptide motif at the base of the gp120 V3-loop ${ }^{25,32,42,45}$. bNAbs against the V3/N332 $2_{\mathrm{gp} 120}$ site isolated from several HIV1 -infected donors can adopt different Env-binding orientations to engage the conserved epitope $e^{29,44}$ and display a wide array of interactions with surrounding glycans, including glycans at positions $\mathrm{N} 301_{\mathrm{gp} 120}$ (the PGT128 bNAb), N137 gp120 $/ \mathrm{N} 156_{\mathrm{gp} 120} /$ N301 ${ }_{\text {gp120 }}$ (PGT121/10-1074 family), and N386 $6_{\text {gp } 120} / \mathrm{N} 392_{\text {gp } 120}$ (PGT135). While under-processed $N$-glycans presenting as a oligomannose patch predominate in the V3/N332 $2_{\text {gp } 120}$ epitope, the recent structure of 10-1074 bound to a natively glycosylated BG505 SOSIP trimer included complex-type $N$-glycans at positions $\mathrm{N} 156_{\mathrm{gp} 120}$ and $\mathrm{N} 301_{\mathrm{gp} 120}{ }^{25}$, and sialic acid-bearing complex-type glycans at the $\mathrm{N} 156_{\mathrm{gp} 120}$ position were shown to be critical for maturation of the CAP256 V2 apex bNAb lineage ${ }^{46}$. Thus, providing structural information of bNAbs bound to Env trimers that include both complex-type and high mannose glycans will facilitate developing strategies for improving bNAb breadth and for design of high-affinity germline-binding immunogens.

BG18 exhibits the highest potency among the V3/N332 gp120 bNAbs described to date ${ }^{47}$. Isolated from an elite controller infected with clade B HIV-1, BG18 displays a similar breadth of coverage ( $64 \%)$ across a panel of HIV-1 strains to the PGT121/ 10-1074 family bNAbs (Supplementary Figure 1), but BG18 neutralizes these strains with a geometric mean $\mathrm{IC}_{50}$ value of 0.03 $\mu \mathrm{g} / \mathrm{mL}$, a higher potency than 10-1074 and other bNAbs in human clinical trials ${ }^{8}$. The structure of unliganded BG18 Fab ${ }^{47}$ exhibited a cleft between the CDRH2 and CDRH3 loops and a long CDRH3 loop that forms a two-stranded $\beta$-sheet as previously observed for PGT121 and 10-1074 Fab structures solved in the absence of HIV-1 Env ${ }^{40}$. However, comparison of the BG18 Fab structure (PDB 5UD9 (https://doi.org/10.2210/ pdb5UD9/pdb)) with structures of unbound PGT121 and 101074 Fabs (4FQ1 (https://doi.org/10.2210/pdb4FQ1/pdb) and 4FQ2 (https://doi.org/10.2210/pdb4FQ2/pdb)) demonstrated structural differences including: (1) a displaced, shorter, and more compact CDRH3 stabilized by a network of hydrogen bonds, (2) differences in variable light $\left(\mathrm{V}_{\mathrm{L}}\right)$ domain orientation relative to the variable heavy $\left(\mathrm{V}_{\mathrm{H}}\right)$ domain, and (3) a second cleft in the antigen-binding site between CDRH3 and CDRL1/CDRL3. In addition, single-particle electron microscopy showed that BG18 exhibited a different orientation compared with PGT121/10-1074 and other V3/N332 ${ }_{\text {gp } 120}$ bNAbs for binding HIV-1 Env ${ }^{47}$.

To better understand the molecular mechanism underlying BG18's interactions with Env glycans and increased potency compared with other $\mathrm{V} 3 / \mathrm{N} 332_{\text {gp } 120}$ bNAbs, we determined the crystal structures of natively glycosylated clade A (BG505) and clade B (B41) Envs in complex with BG18 Fab. We used the increased brightness of an X-ray free electron laser ${ }^{48}$ (XFEL) to circumvent the limitations of crystal size and improve the resolution to $3.8 \AA$ for our BG18-BG505-35O22 complex. We found that BG18 binds the V3/N332 ${ }_{\text {gp } 120}$ glycan site in a distinct manner relative to PGT121-like bNAbs, primarily through rearrangements in its $\mathrm{V}_{\mathrm{H}}$ and $\mathrm{V}_{\mathrm{L}}$ domains. Analysis of BG18 interactions bound to a natively glycosylated Env showed engagement with oligomannose glycans near the base of the V3-loop and the conserved GDIR peptide motif. Moreover, BG18's CDRL1 and CDRL2 formed part of a cleft within $8 \AA$ of gp120's variable loop 1 (V1-loop), increasing BG18's protein surface contact with Env. These structures provide valuable information for understanding the promiscuity of $\mathrm{V} 3 / \mathrm{N} 332_{\mathrm{gp} 120}$ glycan-directed bNAbs that will facilitate current efforts to evaluate them for therapeutic use in HIV-1-infected humans ${ }^{8}$ and to target their epitope for immunogen design ${ }^{49,50}$.

\section{Results}

Structures of natively glycosylated Env-BG18 complexes. Structural insight into BG18 binding in the context of a natively glycosylated Env was achieved by crystallizing bNAb Fabs complexed with Env trimers expressed and purified from Chinese hamster ovary $(\mathrm{CHO})$ cells ${ }^{51}$, which include similar glycoforms as Env trimers expressed in human cell lines ${ }^{52}$. Our crystals comprised natively glycosylated Env SOSIP.664 trimers from clade A (BG505) or clade B (B41) strains bound to Fabs from BG18 and either 35O22, a gp120-gp41 interface-spanning $\mathrm{bNAb}^{53}$, or IOMA, a VH1-2 CD4bs bNAb ${ }^{25}$. We solved the structures of BG18-BG505-35O22, BG18-B41-35O22, and BG18-BG505-IOMA complexes to resolutions of 4.1, 4.9, and $6.7 \AA$, respectively (Fig. 1 and Table 1 ) by molecular replacement (Methods) using synchrotron radiation datasets. Subsequently, 
a
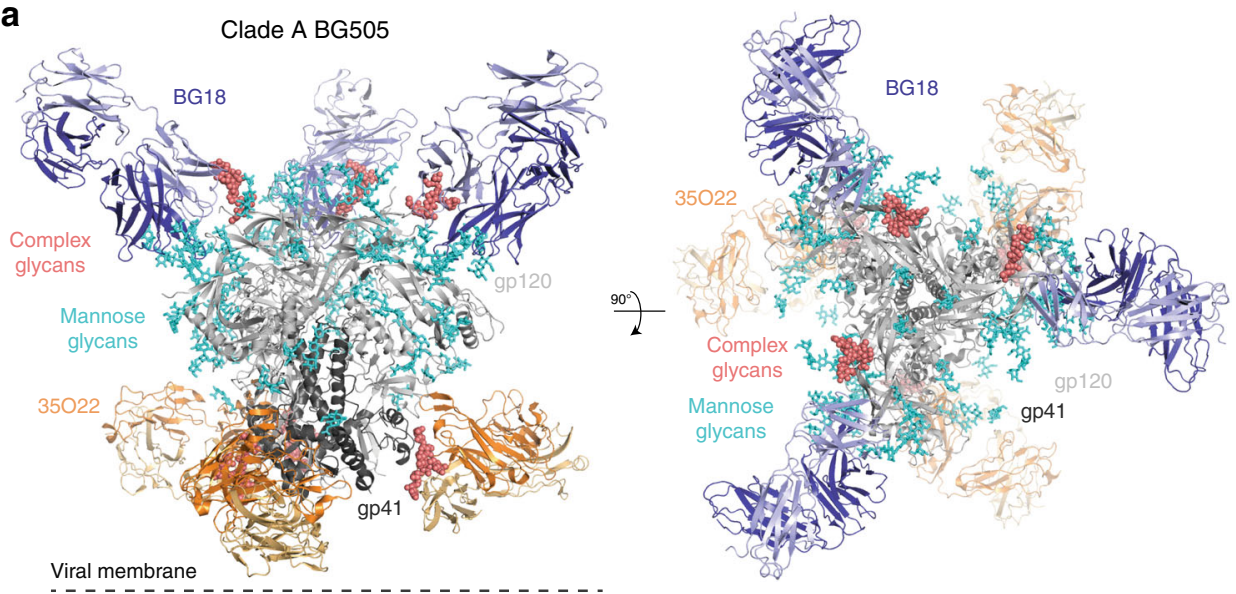

b $----$

C

d
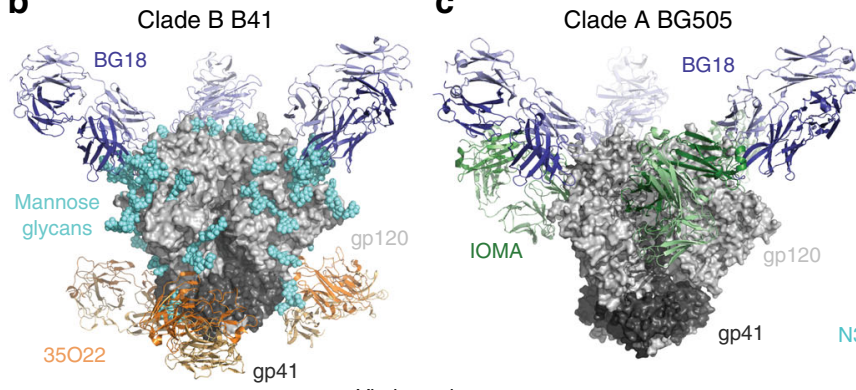

Viral membrane

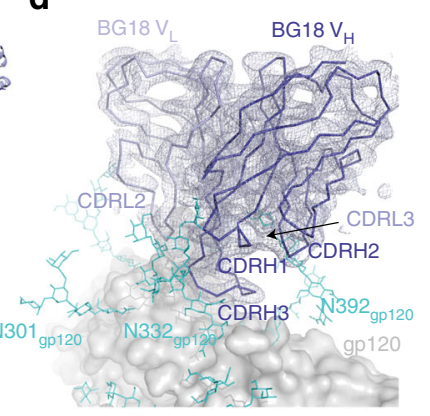

Fig. 1 Crystal structures of natively glycosylated HIV-1 Env trimers complexed with BG18. a Cartoon representation of the $3.8 \AA$ structure of BG505 Env (gp120, light gray; gp41, dark gray) in complex with BG18 (blue) and $35 \mathrm{O} 22$ (orange) Fabs. Ordered, native high-mannose glycans (cyan) are represented as sticks, and complex-type glycans (salmon) are shown as spheres. b $4.9 \AA$ structure of B41 Env bound to BG18 (blue) and 35022 (orange). Glycans shown as cyan spheres. c $6.7 \AA$ structure of BG505 Env bound to BG18 (blue) and IOMA (green) Fabs. Glycans were not modeled due to the limited resolution. $\mathbf{d}$ Close-up of the $B G 18$ binding site $\left(V_{H}\right.$ in dark blue, $V_{L}$ in light blue superimposed on the final $2 F_{0}-F_{\text {calc }}$ electron density map contoured at $\left.1.2 \sigma\right)$ on the surface of gp120 (gray) from the BG18-BG505-35022 structure. Ordered glycans (cyan) near the BG18 binding site are represented as sticks

XFEL diffraction data collected by manually targeting individual crystals $(\sim 75 \mu \mathrm{m} \times 75 \mu \mathrm{m} \times 50 \mu \mathrm{m}$ dimensions $)$ improved intensities of high-angle Bragg reflections (Supplementary Figure 2) as previously observed for XFEL datasets ${ }^{48,54}$, resulting in a $3.8 \AA$ resolution structure of the BG18-BG505-35O22 complex from 520 still diffraction images (Fig. 1a). The use of B-factor sharpening ${ }^{55}$ in our BG18-BG505-35O222 structures permitted visualization and refinement of most amino acid side chains, particularly at the Fab-Env interface.

The BG18-BG505-35O22, BG18-B41-35O22, and BG18BG505-IOMA structures each comprised an Env trimer bound to three BG18 Fabs and three $35 \mathrm{O} 22$ or IOMA Fabs (Fig. 1a-c), with ordered electron density corresponding to native glycans at the Fab interfaces (Fig. 1d and Supplementary Figure 3a). The orientations of BG18 Fab with respect to gp120 were preserved across the three crystal structures and a low-resolution EM structure of a BG18-BG505 complex ${ }^{47}$ (Supplementary Figure $3 \mathrm{~b}-\mathrm{d}$ ), indicating that the orientation was conserved across different Env strains and was not an artifact of crystal packing (Supplementary Figure 3e,f). In addition, BG18 did not alter binding modes at the gp41/gp120 interface and CD4bs by $35 \mathrm{O} 22$ or IOMA, respectively, as these Fabs adopted similar conformations as previously observed on trimeric Envs ${ }^{25,27}$.

Superimposition of the BG18 $\mathrm{V}_{\mathrm{H}}-\mathrm{V}_{\mathrm{L}}$ coordinates in the BG18-BG505-35O22 structure with $\mathrm{V}_{\mathrm{H}^{-}} \mathrm{V}_{\mathrm{L}}$ in unbound BG18 Fab (PDB 5UD9 (https://doi.org/10.2210/pdb5UD9/pdb)) resulted in a $1.3-\AA$ root mean square deviation (rmsd) $(240 \mathrm{Ca}$ atoms), demonstrating that BG18 CDR loops (except for CDRL2, which was disordered in unbound $\mathrm{BG} 18^{47}$ ) did not undergo large conformational rearrangements upon binding Env, and thereby maintained the previously observed clefts (Fig. 2a, b). Most notably, the conformation and location of CDRH3 in unbound BG18, which differs from CDRH3s in unbound PGT121 and $10-1074^{40,47}$, were preserved in the Env-bound BG18 structure (Fig. 2c, d). Additionally, interactions with gp120 and glycans at the base of the V3-loop resulted in stabilization of CDRL2.

BG18 adopts a distinct Env-binding orientation. A lowresolution Env-bound BG18 structure derived by negative stain EM showed an orientation for BG18 distinct from the orientations of $10-1074$ and other $\mathrm{V} 3 / \mathrm{N} 332_{\mathrm{gp} 120} \mathrm{bNAbs}^{47}$. Despite crystallization contacts involving Fabs (Supplementary Figure 3e, f), BG18 maintained this distinct orientation in our crystal structures compared with orientations in structures of HIV-1 Env trimers with Fabs from 10-1074, PGT122, a PGT121 intermediate, and PGT124 $25,27,31,50$ (Fig. 3 and Supplementary Figure 4). To analyze these differences, we calculated the rotation and translation for $\mathrm{V}_{\mathrm{H}}-\mathrm{V}_{\mathrm{L}}$ domains of Env-bound BG18 Fab when compared to Env-bound 10-1074 Fab structures. We found that the mature $\mathrm{BG} 18 \mathrm{~V}_{\mathrm{H}}-\mathrm{V}_{\mathrm{L}}$ domains differed by $\sim 78^{\circ}$ relative to the orientations of 10-1074 Fab $\mathrm{V}_{\mathrm{H}^{-}} \mathrm{V}_{\mathrm{L}}$ domains (Fig. 3d, e), which contrasts with the $\sim 5^{\circ}$ difference between Env-bound PGT122 and 10-1074 Fab orientations. Notwithstanding its different orientation, BG18, 10-1074, and PGT121-like bNAbs share a common mode of interaction with the $\mathrm{N} 332_{\text {gp } 120}$ glycan 
Table 1 Data collection and refinement statistics ${ }^{a, b, c}$

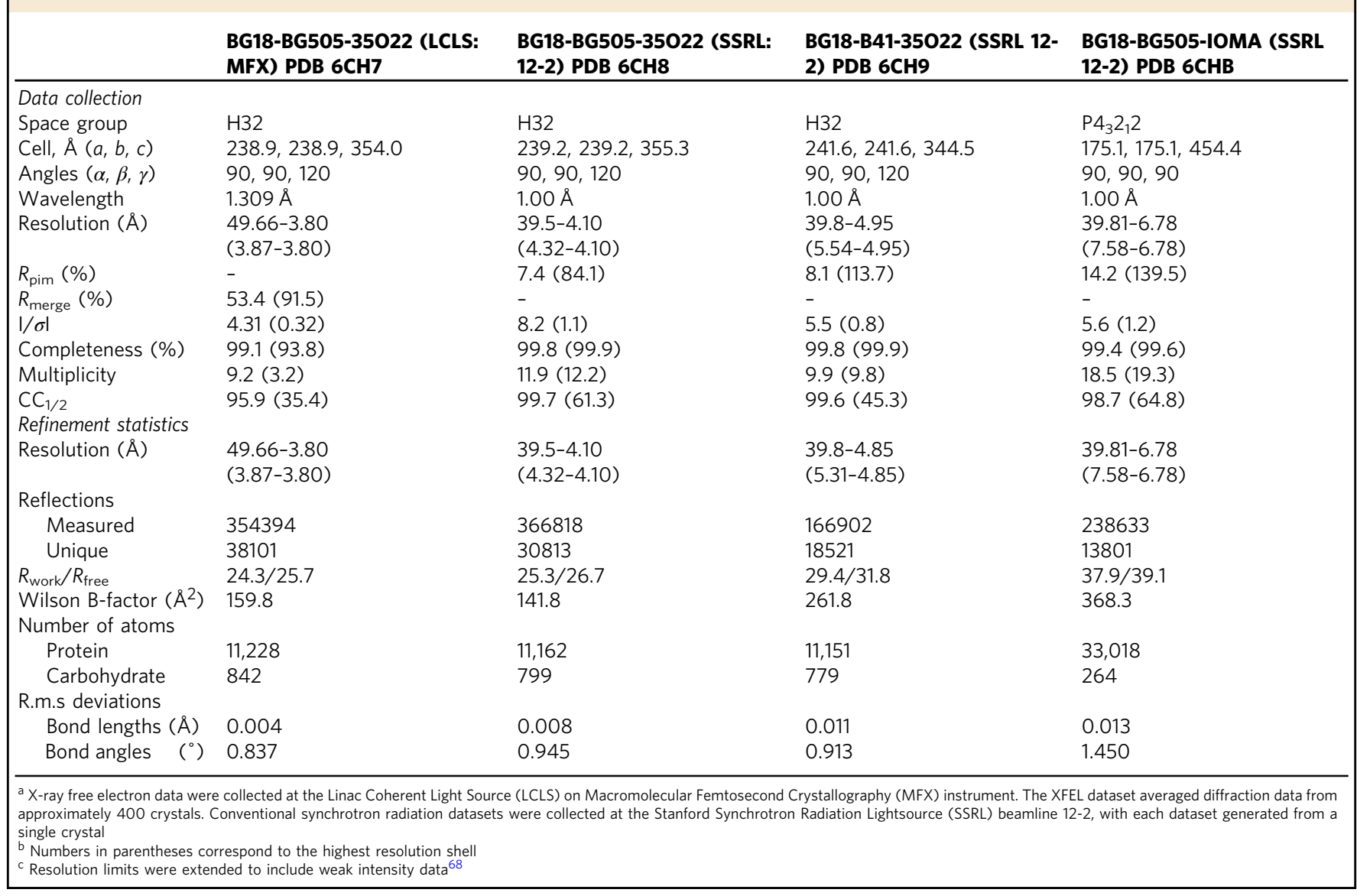

through their CDRH3 loops (Fig. 3c). This interaction includes a structural motif with a consensus R-I-Y-G-V/I-I sequence (BG18 residue numbers 101-106 and 10-1074/PGT122 residue numbers 100-100E; Fig. 2d) encoded by the same antibody D3-3 gene segment, likely explaining the nearly identical $\mathrm{N} 332_{\text {gp120 }}$ glycan recognition. Given that $\mathrm{CDRH} 3$ is a primary determinant of the interactions of V3/N332 ${ }_{\text {gp } 120}$ bNAbs with Env trimer ${ }^{29,32,40,44}$, its displacement in both unbound and Env-bound BG18 (Fig. 2c) rationalizes its orientation of Env binding relative to other V3/ N332 gp120 bNAbs. Consistent with these observations, BG18's footprint at the V3/N332 ${ }_{\text {gp } 120}$ epitope differs from PGT121/101074-like bNAbs, such that its interactions with Env glycans and protein components are mediated by different CDR loops relative to the CDR loops used by 10-1074 (Fig. 4a, b and Supplementary Figure 5).

$N$-linked glycan interpretation and interactions with BG18. Modeling of glycans was achieved in our structures by interpreting electron density at PNGSs using $2 F_{\mathrm{o}}-F_{\mathrm{c}}$ maps calculated with model phases and in composite annealed omit maps to reduce model bias ${ }^{56}$. In the $\mathrm{BG} 18-\mathrm{BG} 505-35 \mathrm{O} 22$ structures, $17 \mathrm{~N}$ linked glycans were modeled into ordered electron density, forming glycan arrays that extended $\sim 22 \AA$ from the Env surface (Fig. 1a and Supplementary Figure 6). N-glycans near the BG18 and $35 \mathrm{O} 22$ interfaces were modeled as mostly oligomannose $\left(\mathrm{Man}_{5-9} \mathrm{GlcNAc}_{2}\right)$ in our structures to avoid over-interpretation of the electron density, except for complex-type glycans at positions $\mathrm{N} 88_{\mathrm{gp} 120}$ and $\mathrm{N} 156_{\mathrm{gp} 120}$, which were assigned as complex-type based on density for a core fucose in our $3.8 \AA$ XFEL structure (Supplementary Figure 7). Although complex- type $N$-glycans were modeled at positions $\mathrm{N} 160_{\text {gp } 120}, \mathrm{~N} 276_{\text {gp } 120}$, $\mathrm{N} 301_{\mathrm{gp} 120}$, and $\mathrm{N} 392_{\mathrm{gp} 120}$ in a previous natively glycosylated 10-1074-Env-IOMA structure ${ }^{25}$ and observed at positions $\mathrm{N} 160_{\text {gp } 120}, \mathrm{~N}_{197} 7_{\text {gp120, }}$, and $\mathrm{N} 276_{\text {gp120 }}$ by mass spectrometry ${ }^{8,52,57}$, our datasets did not show characteristic densities for complex glycans at these positions. Modeling of oligomannose $\mathrm{N}$-linked glycans into ordered electron density was also possible in the $4.9 \AA$ BG18-B41-35O22 structure, and for the $\mathrm{N} 332_{\mathrm{gp} 120}$ glycan in the $6.7 \AA$ BG18-BG505-IOMA structure (Fig. 1b,c and Supplementary Figure 6). Overlay of BG505 and B41 Env structures showed conservation of the glycan shield surrounding the BG18 binding site (Supplementary Figure 6). Despite resolution limitations that necessitated glycan modeling as predominantly oligomannose at PNGSs shown to attach complex glycoforms, the use of natively glycosylated Env trimers in the crystallization complexes ensured accuracy of bNAb binding orientations and interfaces with Env trimer.

As seen in other PGT121-like bNAb-Env SOSIP structures $25,27,31,50$, BG18's primary interaction was with the $\mathrm{N} 332_{\text {gp120 }}$ glycan $\left(\mathrm{Man}_{9} \mathrm{GlcNAc}_{2}\right)$, which interfaced with CDRH3, CDRH1, and CDRL2 (Fig. 4a,c; $1170 \AA^{2}$ total buried surface area (BSA)). This contrasts with PGT121-like bNAb interactions that pack the $\mathrm{N} 332_{\text {gp120 }}$ glycan into a groove between CDRH3 and CDRL1/2 25,29,31 (Fig. 4b). Indeed, the observed differences in the orientation of the BG18 Fab light chain on Env influenced the $\mathrm{N} 332_{\text {gp } 120}$ glycan conformation, as the glycan D1 arm reached in close proximity to CDRL2 and the gp120 GDIR motif in the BG18-BG505 complex (Fig. 4c), which differs from the interactions of the $\mathrm{N} 332_{\text {gp } 120}$ glycan D1 arm with light chain framework region 3 (FWRL3) in the 10-1074-BG505 complex 
a

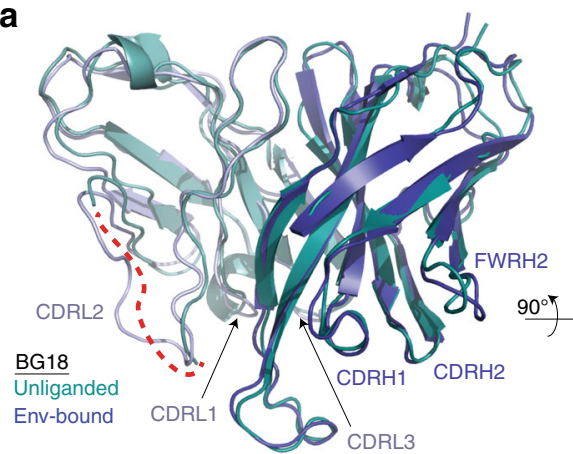

c

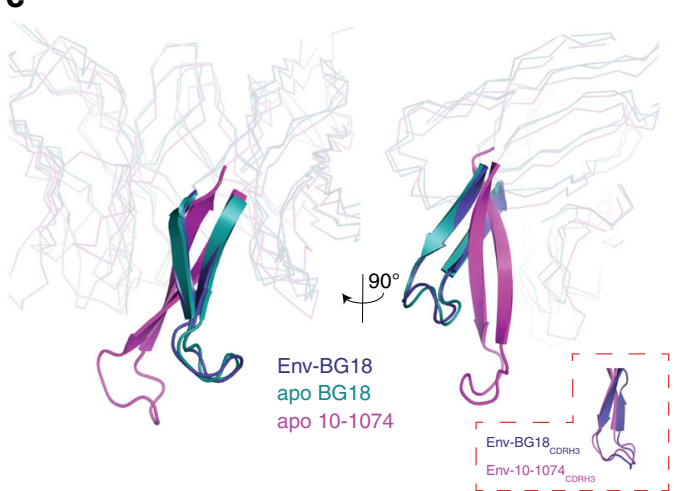

b

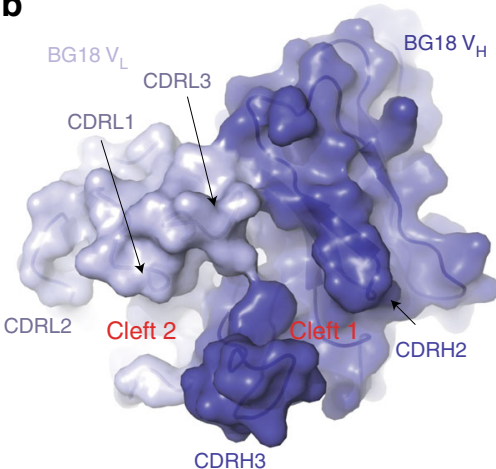

d

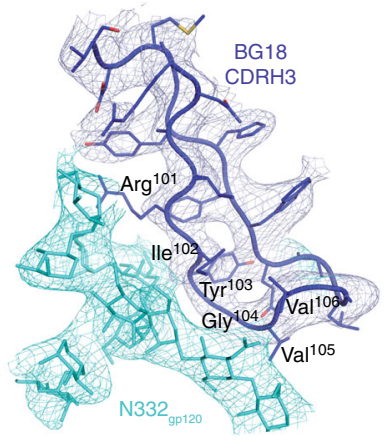

Fig. 2 Comparison of apo- and Env-bound BG18 variable domains. a Superposition of $\mathrm{V}_{\mathrm{H}^{-}} \mathrm{V}_{\mathrm{L}}$ domains (230 $\mathrm{C} \alpha$ atoms) of unliganded BG18 (deep teal; PDB 5UD9 (http://dx.doi.org10.2210/pdb5UD9/pdb)) with BG18 in the BG18-BG505 structure (blue) showed conservation of the BG18 antigen-binding site and ordering of CDRL2 in the BG505-bound structure (dashed red line represents disordered CDRL2 in unliganded BG18). b Surface representation of BG18 in the BG505-bound structure showed conservation of BG18 clefts, including the cleft between CDRH3 and CDRL1/3 loops observed in the BG18 apo structure ${ }^{47}$. $\mathbf{c}$ Ribbon and cartoon representation of the overlay between BG18 (aligned on the Fab $\mathrm{V}_{\mathrm{H}}$ domains) in the BG18-BG505-35O22 complex (blue), unliganded BG18 (deep teal), and unliganded 10-1074 (magenta; PDB 4FQ2 (http://dx.doi.org10.2210/pdb4FQ2/pdb)). CDRH3 loops for the three structures are represented as cartoons. Inset: overlay of BG18-BG505 and 10-1074-BG505 (PDB 5T3Z (http://dx.doi.org10.2210/pdb5T3Z/pdb)) CDRH3 loops. d Cartoon and stick representation of BG18 CDRH3 recognition of the N332 gp120 glycan. BG18 residues comprising a conserved structural motif (R-I$\mathrm{Y}-\mathrm{G}-\mathrm{V} / \mathrm{I}-\mathrm{I})$ are labeled. Electron density contoured at $1 \sigma$ from $2 F_{\mathrm{obs}}-F_{\text {calc }}$ composite annealed omit map calculated with phases from models with the N332 gp120 glycan and BG18 CDRH3 coordinates omitted to reduce potential phase bias (cyan and blue mesh)

(Fig. 4b). Modeling of Env-bound 10-1074 Fab conformation onto the gp120 subunit from the BG18-BG505-35O22 structure showed clashes between the $\mathrm{N} 332_{\text {gp } 120}$ glycan and the CDRL1/2 loops and FWRL3 of 10-1074 (Fig. 4d), suggesting that the distinct BG18 interaction with Env stabilizes a N332 gp120 glycan conformation that is not possible when binding PGT121/101074-like bNAbs ${ }^{29,31}$.

In addition to the BG18 interaction with the $\mathrm{N} 332_{\text {gp120 }}$ glycan, BG18 makes secondary interactions with the $\mathrm{N} 392_{\text {gp } 120}$,

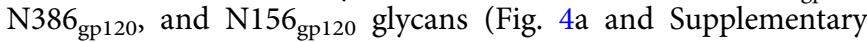
Data 1). These interactions are in contrast with PGT121-like bNAbs that interact with the $\mathrm{N} 137_{\mathrm{gp} 120}, \mathrm{~N} 156_{\mathrm{gp} 120}$ glycans in the V1-loop, and $\mathrm{N} 301_{\mathrm{gp} 120}$ glycans at the base of the V3-loop (Fig. 4b and Supplementary Data 1$)^{25,27,32}$. When comparing the BG18-Env interaction with the only other structure of a V3/ $\mathrm{N} 332_{\text {gp120 }}$ bNAb bound to natively glycosylated Env (10$1074-\mathrm{BG} 505)^{25}$, the rearrangement of BG18's $\mathrm{V}_{\mathrm{L}}$ domain reduced BG18's contact with the $\mathrm{N} 301_{\text {gp120 }}$ glycan (modeled as a core pentasaccharide in the BG18-BG505-35O22 structure) compared to $10-1074$ 's contacts with the $\mathrm{N} 301_{\mathrm{gp} 120}$ glycan (modeled as complex-type biantennary in the 10-1074-BG505-IOMA structure; PDB 5T3X (https://doi.org/10.2210/pdb5T3X/pdb)) (Fig. 4a, b). Interestingly, $\mathrm{V}_{\mathrm{L}}$ domain rearrangements allowed BG18 engagement of the $\mathrm{N} 392_{\text {gp } 120}$ glycan through its CDR loops (Fig. 4a). The N392 ${ }_{\text {gp120 }}$ glycan threads between the BG18 cleft that is located between CDRH3 and CDRL1/L3 ${ }^{47}$ (Fig. 2b), burying $\sim 425 \AA^{2}$ of Fab surface area against the glycan. This interaction resembles the PGT135 interaction with monomeric gp120 in which $\sim 550 \AA^{2}$ of gp120 surface area is buried against the $\mathrm{N} 392_{\text {gp120 }}$ glycan $^{44}$. However, in contrast to PGT135, which does not neutralize strains lacking the $\mathrm{N} 392_{\mathrm{gp} 120}$ or $\mathrm{N} 386_{\mathrm{gp} 120}$ glycans, BG18 remains potent against strains lacking glycans at these positions, showing only a $\sim 4$-fold reduction in potency (Table 2). Moreover, binding affinities determined by surface plasmon resonance (SPR) showed no effect when the $\mathrm{N} 392_{\mathrm{gp} 120}$ glycan was removed from BG505 SOSIP (Supplementary Figure 8). These results are comparable to PGT121-like bNAbs, which equivalently neutralize strains $+/-$ the $\mathrm{N} 392_{\mathrm{gp} 120}$ and N386 gp120 glycans (Table 2), suggesting that BG18 combines binding and neutralization properties of both PGT121-like and PGT135-like bNAbs.

Molecular details of BG18-Env interactions. BG18 conserves interactions with the gp120 GDIR peptide motif at the base of the V3-loop that are seen in other V3/N332 ${ }_{\text {gp } 120}$ bNAbs (Fig. 5a, b and Supplementary Figure 5). However, rotation of BG18's light chain relative to other $\mathrm{V} 3 / \mathrm{N} 332_{\mathrm{gp} 120}$ bNAbs places only CDRL2 in close proximity to the gp120 GDIR peptide motif, compared to engagement of GDIR by multiple CDR loops and framework 

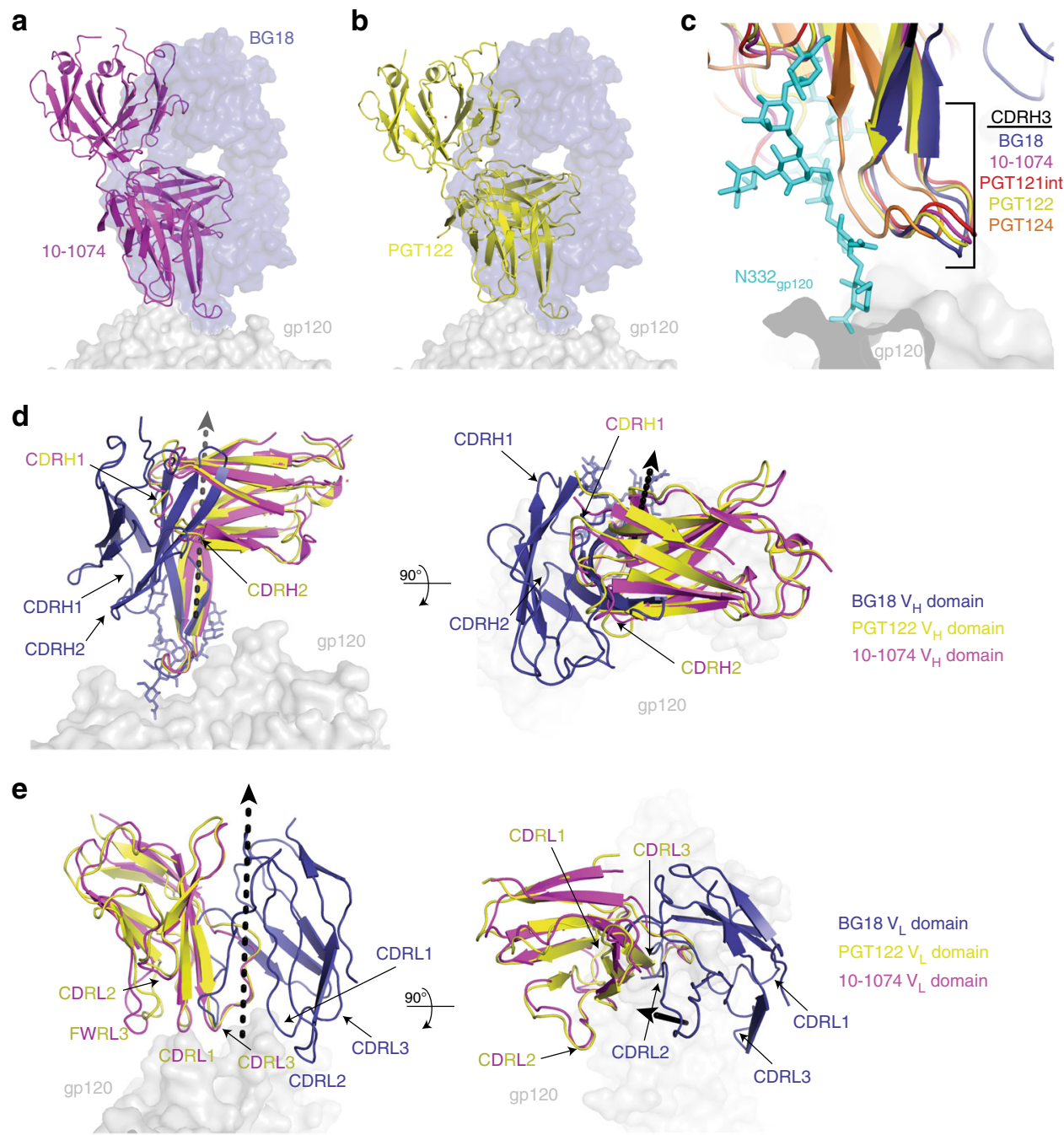

Fig. 3 Distinct orientation on HIV-1 Env of BG18 compared to other V3/N332 $2_{\text {gp120 }}$ bNAbs. BG18 Fab (blue, surface) orientation on gp120 (gray, surface) overlaid with 10-1074 (a, magenta; PDB 5T3Z (http://dx.doi.org10.2210/pdb5T3Z/pdb)) and PGT122 (b, yellow; 5FYL (http://dx.doi.org10.2210/ pdb5T3Z/pdb)) and PGT122 (b, yellow; 5FYL (http://dx.doi.org10.2210/pdb5FYL/pdb)) Fabs. c Overlay of CDRH3 loop of BG18 (blue), 10-1074 (magenta), PGT122 (yellow), PGT121 precursor (red; PDB 5CEZ (http://dx.doi.org10.2210/pdb5CEZ/pdb)), and PGT124 (orange; PDB 4R2G (http://dx.doi.org10.2210/pdb4R2G/pdb)) after alignment of bound gp120s (gray, surface) demonstrating a conserved structural motif recognizing the N332 gp120 glycan (cyan). d, e Comparison of $\mathrm{V}_{\mathrm{H}}-\mathrm{V}_{\mathrm{L}}$ domains of PGT122 (yellow) and 10-1074 (magenta) with BG18 (blue). The $\mathrm{V}_{\mathrm{H}}-\mathrm{V}_{\mathrm{L}}$ domain orientation of BG18 on Env trimer is related by a $78^{\circ}$ rotation and $6.8 \AA$ translation about the indicated axis (black dashed arrow) to the 10-1074 variable domains after alignment against gp120 in the BG18-Env and 10-1074-Env structures

regions observed in 10-1074/PGT121-like bNAb recognition (Fig. $5 \mathrm{~b}, \mathrm{c})^{25,29,31}$. These differences in light chain interactions reflect the germline origins of the BG18 and PGT121/10-1074 light chains, which derive from different VL gene segments (Fig. 5d). However, in an example of convergence toward a common chemical binding mechanism, light chain residues involved in GDIR recognition by BG18 CDRL2 derive from hypermutation from the germline LV3- $25^{\star} 02$ gene segment, whereas serines in the CDRL3s of PGT121 and 10-1074 derive from $J$ regions chosen during $\mathrm{V}-\mathrm{J}$ joining.

B41 Env harbors a GNIR sequence instead of GDIR, allowing us to analyze the $\mathrm{D} 325 \mathrm{~N}_{\mathrm{gp} 120}$ substitution in our BG18-B41$35 \mathrm{O} 22$ structure. Side chain placement was not possible due to low resolution (4.9 $)$ ), but the BG18 interaction with B41 resembled its interaction with BG505 in the BG18-BG505-35O22 structure (Supplementary Figure 9), suggesting that BG18 recognizes the GNIR motif analogously to how it recognizes GDIR. Consistent with this result, analysis of BG18 neutralization of HIV-1 isolates containing GNIR motifs showed only a 2-fold loss in potency, by contrast to PGT121 or PGT122, which showed $\sim 6$-fold and $\sim 44$-fold losses, respectively (Table 2). However, while BG18 S53 ${ }_{\mathrm{LC}}$ potentially engages $\mathrm{N} 325_{\mathrm{gp} 120}$ (Supplementary Figure 8b), the loss of BG18 Q54 $4_{\mathrm{LC}}$ contacts resulted in the disorder of CDRL2 residues 54-60 in our BG18-B41 structure.

Further comparisons between BG18-Env and 10-1074-Env bound structures showed that BG18 CDR loops and light chain framework region 3 are located in close proximity to the gp 120 V1-loop (residues 126-158, Supplementary Figure 5), of which residues 140-150 were disordered in the BG18-Env structures (Fig. 6a) and other Env structures ${ }^{22,25,27,31}$. Despite variability in V1 across HIV-1 Envs, we observed engagement of V1-loop protein components with BG18 CDRL2 and FWRL3 residues (Fig. 6b), accommodating the V1-loop into the nearby positively charged cleft formed by CDRH3, CDRL1/L2, and FWRL3 loops (Fig. 6b, c). The electronegativity of this cleft potentially increases protein-protein interactions with BG505 and other HIV-1 strains harboring polar or acidic residues in this region (Fig. 6c). Although PNGSs are commonly found in the V1-loop, the 
a

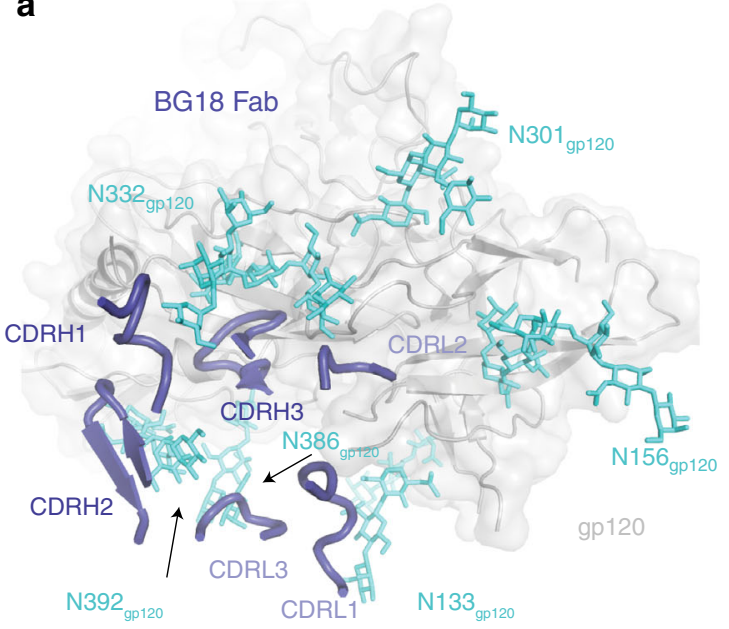

b

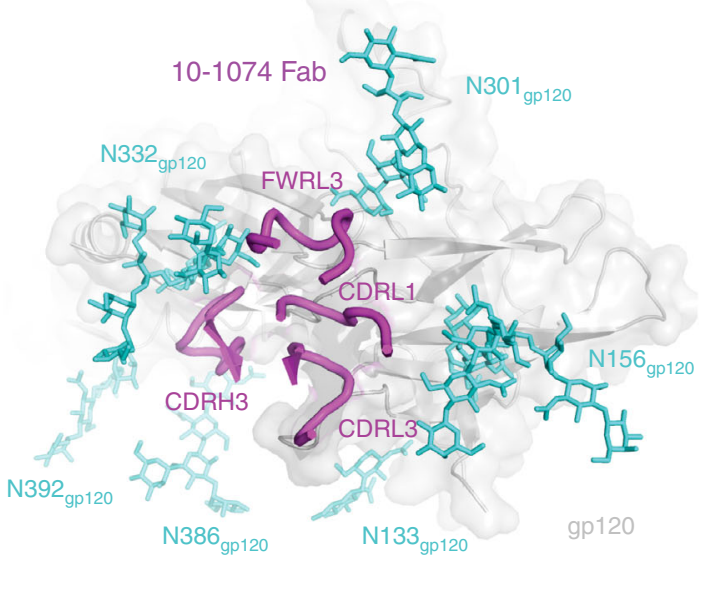

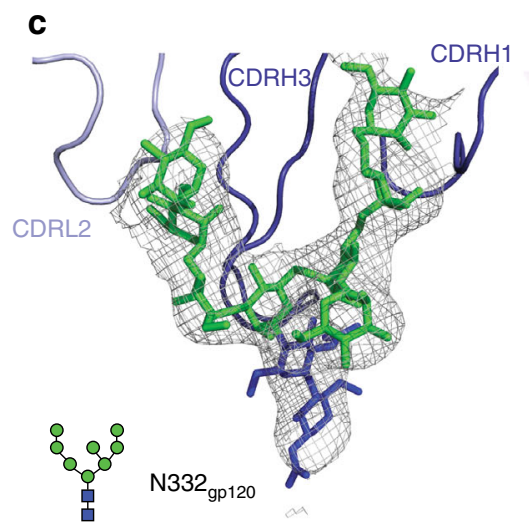
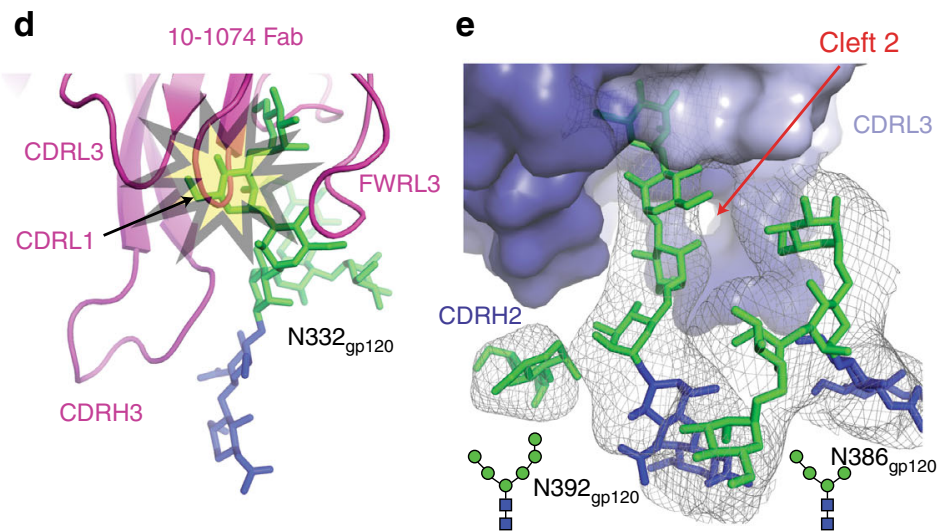

Fig. 4 Glycan interactions with V3/N332 ${ }_{\text {gp120 }}$ bNAbs in structures including natively glycosylated Env. a, b Comparison of the orientations on gp120 (gray surface and cartoon) of the CDR loops from (a) BG18 (blue, ribbon) and (b) 10-1074 (magenta, ribbon) demonstrating that the BG18 variable domains are rotated clockwise about $\mathrm{CDRH} 3$ relative to the 10-1074 variable domains. The distinct BG18 orientation on gp120 resulted in contacts with $\mathrm{N} 156_{\mathrm{gp} 120}$

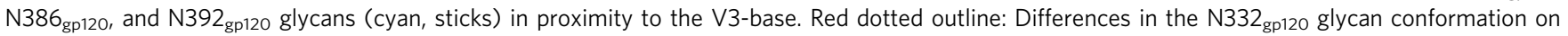
BG505 Env bound to BG18 (a) or to 10-1074 (b). c Close-up of BG18 interaction with the N332 gp12o glycan showing CDRH3, CDRH1 and CDRL2 loops at the glycan interface. d Overlay of BG18-BG505 and 10-1074-BG505 (PDB 5T3Z (http://dx.doi.org10.2210/pdb5T3Z/pdb)) structures showed that the

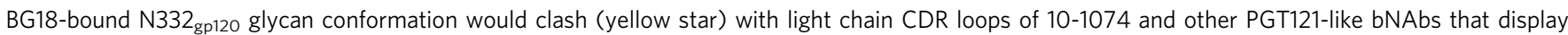
nearly identical binding modes. e Surface representation of BG18 interactions with the N392 gp120 and N386 gp120 glycans showing the N392 $2_{\text {gp120 }}$ glycan buried ( $\sim 800 \AA^{2}$ total BSA) inside cleft 2 located between the CDRH3 and CDRL1/3 loops. The N386 gp120 glycan associates weakly with light chain BG18 ( $45 \AA^{2}$ total BSA), but forms branch-branch interactions with the N392 gp120 glycan. c, e Electron density contoured at $1 \sigma$ from $2 F_{\text {obs }}-F_{\text {calc }}$ composite annealed omit maps calculated with phases from models with glycan coordinates omitted to reduce potential phase bias (gray mesh)

presence of glycans in this region do not notably affect BG18 neutralization (e.g., $\mathrm{N} 133_{\text {gp 120 }}, \mathrm{N} 137_{\text {gp 120 }}$, and $\mathrm{N} 156_{\text {gp } 120}$ glycans; Fig. $6 \mathrm{a}$ and Table 2), despite the roof of the cleft being only $\sim 6-8$ $\AA$ above the V1-loop site. In comparison, the surface of 10-1074 in the comparable region is neutral and does not exhibit a cleft architecture, and only minimally contacts the V1-loop backbone through R94 $4_{\mathrm{LC}}$ (Supplementary Figure 10). Taken together, these observations may explain BG18's increased potency, as BG18 can engage multiple glycan and protein component regions of gp120 (Supplementary Data 1).

\section{Discussion}

Structures of bNAbs complexed with HIV-1 Env trimers have helped elucidate the molecular correlates for anti-HIV-1 antibody breadth and potency. Here we report four crystal structures of the highly potent V3/N332 gp120 bNAb BG18 bound to natively glycosylated clade A and clade B Envs (Fig. 1a-c). Our structures of clade A BG505 and clade B B41 HIV-1 strains represent only the second example of fully and natively glycosylated Env crystal structures, with the first being BG505 complexed with another V3/N332 gp120 bNAb, 10-1074, and with the CD4bs bNAb IOMA $^{25}$. Given the crucial role the Env glycan shield plays in HIV-1's immune evasion strategies ${ }^{13,58}$, the prevalence of bNAbs that interact with complex glycans ${ }^{18,25,58}$, and the importance of complex glycans in bNAb maturation ${ }^{46}$, solving structures containing both complex and high-mannose glycans provides a more complete picture of bNAb recognition of Env epitopes. The newly identified BG18-Env-35O22 crystal lattice system packs solely through Fab interactions (Table 1 and Supplementary Figure 3e), similar to crystals of previously described Env-bNAb complexes ${ }^{25,27}$, thus providing an additional system to study HIV-1 Env diversity. Moreover, the improvement in resolution from $4.1 \AA$ using conventional crystals to $3.8 \AA$ resolution by exposing smaller crystal volumes using an XFEL (Supplementary Figure 2) offers the potential to examine natively glycosylated Env trimer-Fab structures to higher resolutions. The demonstration that two HIV-1 Env trimers (BG505 and B41) can be crystallized in different crystal packing lattices without converting their 


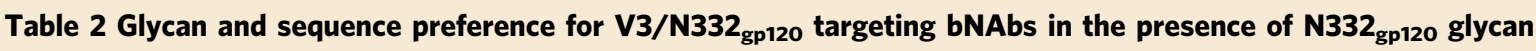

\begin{tabular}{lllll} 
& \multicolumn{1}{l}{$\mathbf{I C}_{\mathbf{5 0}}$ values $(\boldsymbol{\mu g} / \mathbf{m L})^{\mathbf{a}}$} & & \\
\cline { 2 - 4 } & $\mathbf{B G 1 8}$ & $\mathbf{1 0 - 1 0 7 4}$ & $\mathbf{P G T 1 2 2}$ & PGT121 \\
\hline+392 & $0.05(n=67)$ & $0.07(n=65)$ & $0.21(n=88)$ & $0.13(n=224)$ \\
-392 & $0.21(n=13)$ & $0.08(n=201)$ & $0.16(n=16)$ & $0.07(n=65)$ \\
+386 & $0.05(n=67)$ & $0.07(n=234)$ & $0.20(n=90)$ & $0.12(n=255)$ \\
-386 & $0.17(n=13)$ & $0.08(n=32)$ & $0.20(n=14)$ & $0.08(n=34)$ \\
+301 & $0.06(n=78)$ & $0.07(n=262)$ & $0.17(n=99)$ & $0.10(n=283)$ \\
-301 & $0.60(n=2)$ & $0.39(n=4)$ & $12.30(n=5)$ & $12.54(n=6)$ \\
+156 & $0.06(n=73)$ & $0.07(n=253)$ & $0.19(n=97)$ & $0.12(n=276)$ \\
-156 & $0.05(n=7)$ & $0.08(n=13)$ & $0.46(n=7)$ & $0.11(n=13)$ \\
+137 & $0.02(n=9)$ & $0.06(n=55)$ & $0.10(n=15)$ & $0.07(n=60)$ \\
-137 & $0.07(n=71)$ & $0.08(n=211)$ & $0.23(n=89)$ & $0.13(n=229)$ \\
+133 & $0.03(n=18)$ & $0.07(n=65)$ & $0.06(n=23)$ & $0.10(n=70)$ \\
-133 & $0.08(n=62)$ & $0.08(n=201)$ & $0.28(n=81)$ & $0.12(n=219)$ \\
D325 gp120 & $0.05(n=65)$ & $0.05(n=209)$ & $0.11(n=89)$ & $0.07(n=229)$ \\
N325 gp120 & $0.10(n=12)$ & $0.12(n=47)$ & $4.82(n=12)$ & $0.41(n=49)$ \\
\hline
\end{tabular}

a Geometric mean $I_{50}$ values were calculated using HIV Antibody Database ${ }^{74}$, where $n=\#$ of strains. All values were calculated including only strains containing the N332 ${ }_{\text {gpino }}$ PNGS

${ }^{\mathrm{b}}$ Analysis done on the only two strains that met the tested criteria

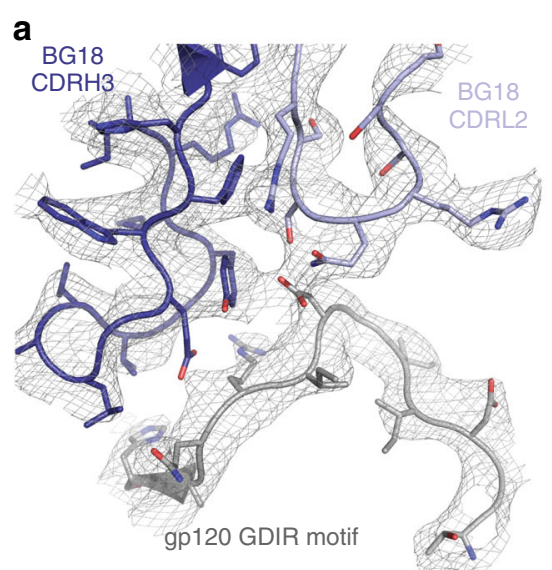

b

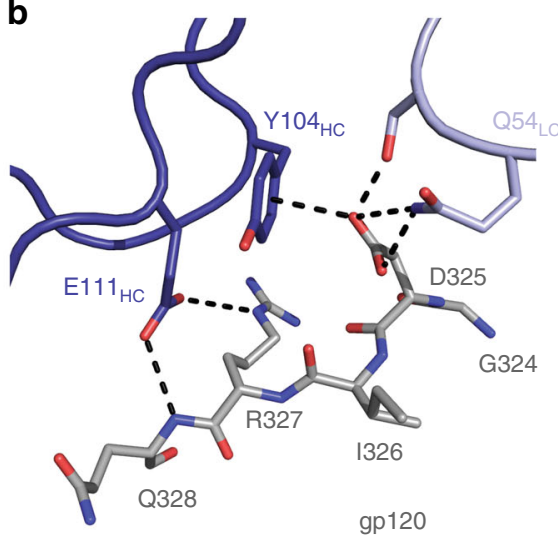

$\underline{\text { CDRL1 }}$

CDRL2

Germline LV3-25*03 SYELTQPPSVSVSPGQTARITCSGDALPKQYAYWYQQKPGQAPVLVIYKDSERPSG I PERFSGSSSGTTVTLTISGVQAEDEADYYCQSADSSG - - - VFGGGTKLTVL

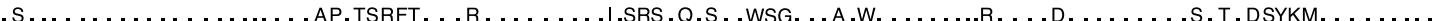

\section{CDRL1}

Germline LV3-21*02 SYVLTQPPSVSVAPGQTARITCGGNNIGSKSVHWYQQKPGQAPVLVVYDDSDRPSGIPERFSGS - - - NSGNTATLTISRVEAGDEADYYCQVWDSSS - - - WVFGGGTKLTVL

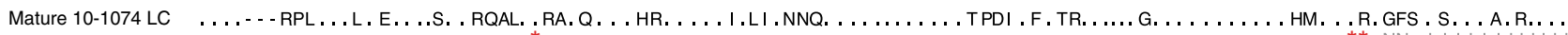

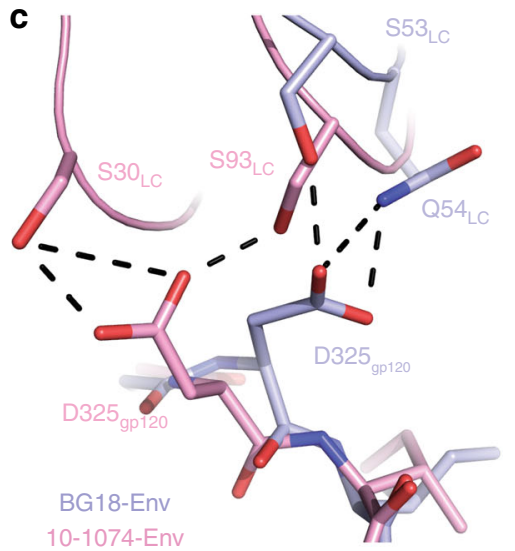

CDRL3

Fig. 5 Comparison of GDIR recognition by BG18 and 10-1074. a Cartoon and stick representation of BG18 CDRH3 (dark blue), BG18 CDL2 (light blue), and gp120 (gray; residues 318-330). Electron density contoured at $1 \sigma$ from $2 F_{\text {obs }}-F_{\text {calc }}$ final refined electron density map with $-100 \AA^{2} B-s h a r p e n i n g$ is shown (gray mesh). b BG18 interactions with the gp120 GDIR motif (G324g $g_{p 120}-D 325 g_{p 120}-1326 g_{p 120}-R 327 g_{p 120}$ ) at the base of the V3-loop. In common with the

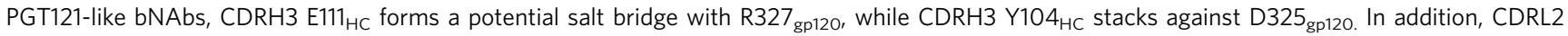

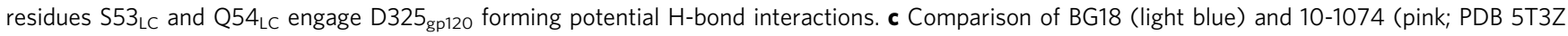

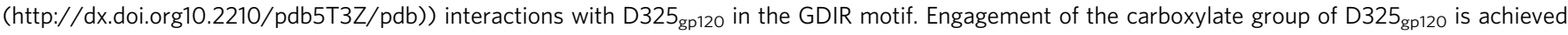
by residues in CDRL2 (BG18) or serine residues in CDRs L1 and L3 (10-1074). Potential H-bonds represented as dashed lines. $\mathbf{d}$ Alignment of sequences of inferred germline and mature light chains of BG18 and 10-1074. The LV3-21*02 germline V gene segment is used for both 10-1074 and PGT121-like antibodies $^{40}$. Red asterisks indicate residues involved in D325 gp120 recognition in the GDIR motif as shown in c

glycans to exclusively high-mannose forms provides an impetus for further crystallization efforts using natively glycosylated HIV-1 Envs. Resulting crystal structures can be compared to natively glycosylated Env structures determined by cryo-EM ${ }^{38}$, a method that does not require crystallization and can therefore be used for heterogeneously glycosylated samples.
Our structures were consistent with previous evidence that BG18 binds with a distinct orientation compared to the prototype PGT121/10-1074 bNAbs in the V3/N332 ${ }_{\text {gp120 }}$ glycan-targeting family ${ }^{47}$ and showed extensive interactions with both protein and glycan components of gp120 (Fig. 1d). BG18's CDRH3 interactions with GDIR and the $\mathrm{N} 332_{\text {gp120 }}$ glycan are conserved with other V3/N332 gp120 bNAbs (Supplementary Figure 5), 
a

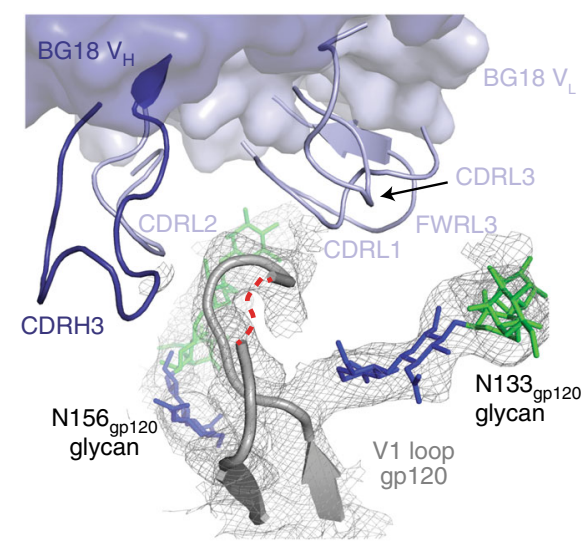

b

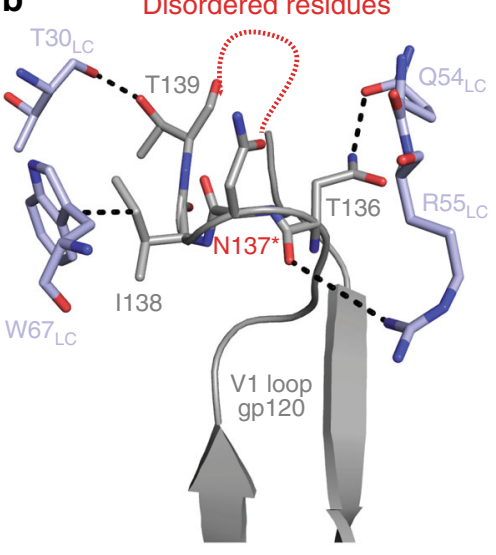

C

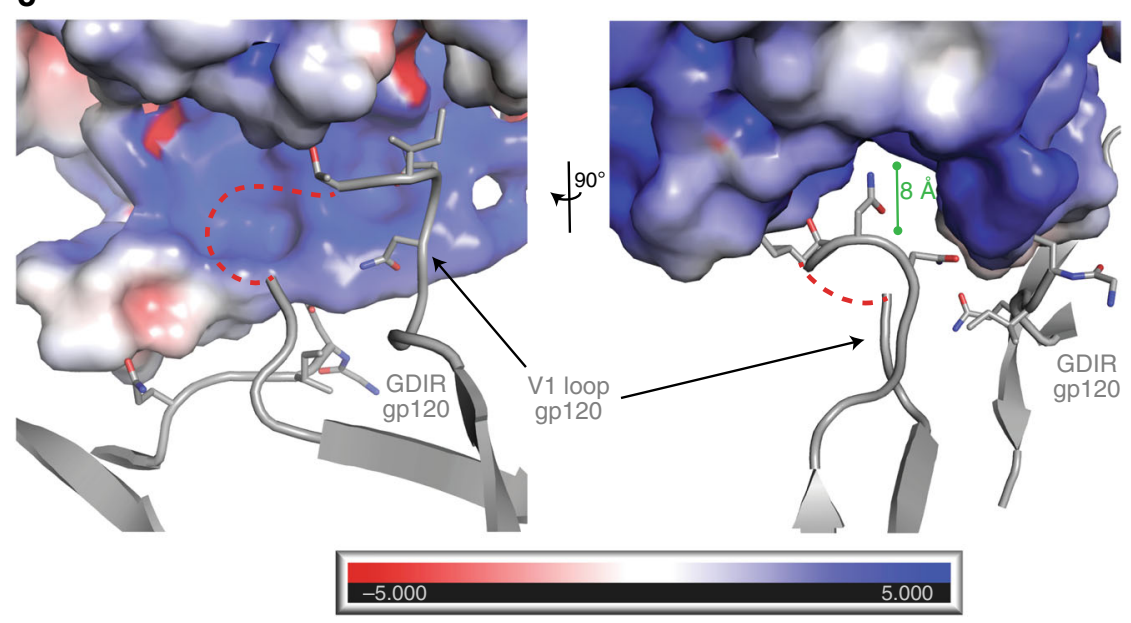

Fig. 6 BG18 contacts with gp120 V1-loop. a Surface and cartoon representation of BG18 $V_{H}$ (dark blue) and $V_{L}$ (light blue) loops involved in gp120 V1-loop (gray; residues 128-158, disordered residues depicted as dashed red line) interactions. Electron density contoured at $1 \sigma$ from a $2 F_{\text {obs }}-F_{\text {calc }}$ composite annealed omit map is shown (gray mesh). Glycans at position N133 gp120 and N156 gp120 are shown. $\mathbf{b}$ Cartoon and stick representation of residue

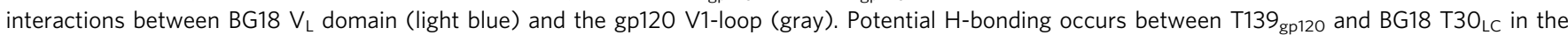
CDRL1 loop. In addition, BG18 W67LC in FWRL3 stacks against 1138 gp120. BG18 contacts with gp120 positions 138 and 139 are likely specific to Envs with V1 characteristics similar to BG505, since similar conformations were not observed in our BG18-B41 structure. $\mathrm{H}$-bonds and pi-stacking are indicated by black dashed lines. Red asterisk on N137 gp120 indicates a PNGS. c Electrostatic surface potentials with red indicating negative electrostatic potential and blue indicating positive electrostatic potential for BG18 shown with cartoon and stick representation of nearby gp120 elements. BG18 includes a positively charged cleft in the vicinity of the gp120 V1-loop (dashed line indicates disordered region), which may provide increased protein-protein interactions with the gp120 surface in HIV-1 strains harboring charged residues in the V1-loop

serving as the main driver for epitope recognition (Figs $3 \mathrm{c}$ and 5b). Strikingly, the D3-3 gene for BG18/10-1074/PGT121 bNAbs that encodes a CDRH3 consensus structural motif responsible for $\mathrm{N} 332_{\text {gp120 }}$ glycan interactions plays a role analogous to the VH1-2 gene for VRC01-like bNAbs ${ }^{24,59,60}$ for epitope targeting. This common feature, which unites the PGT121 and BG18 bNAb families, suggests that it provides the key interaction in the initial binding of their unmutated ancestor precursors to Env. Interestingly, this common interaction occurs despite different orientations for the rest of the $\mathrm{V}_{\mathrm{H}}-\mathrm{V}_{\mathrm{L}}$ domains (Fig. 4a, b). For example, unlike other V3/N332 gp120 bNAbs, BG18's light chain CDR loops straddle the V1-loop of gp120 (Fig. 6), increasing surface contact with gp120 protein components. In addition, BG18's interactions with gp120 N-glycans differ from the PGT121-like family in both engagement and ability to retain potent neutralization properties. For instance, despite extensive interactions with glycans at positions $\mathrm{N} 392_{\mathrm{gp} 120}$ and $\mathrm{N} 386_{\mathrm{gp} 120}$ (Fig. 4a, e and Supplementary Data 1), BG18 can potently neutralize HIV-1 strains lacking these glycans, by contrast to the more weakly neutralizing V3/N332 ${ }_{\text {gp } 120}$ bNAb, PGT135, which relies on $\mathrm{N} 332_{\mathrm{gp} 120}, \mathrm{~N} 392_{\mathrm{gp} 120}$, and $\mathrm{N} 386_{\mathrm{gp} 120}$ glycans for its antiHIV-1 activity ${ }^{44}$ (Table 2). Consistent with this observation, analysis of neutralization by BG18 and PGT121/10-1074 family bNAbs of HIV-1 strains including the N332 $2_{\text {gp } 120}$ glycan, but with and without glycans known to interact with bNAbs targeting the V3/N332 gp120 epitope, showed that BG18 and 101074 retained their anti-HIV-1 potency (mean $\mathrm{IC}_{50} \mathrm{~s}<0.6 \mu \mathrm{g}$ / $\mathrm{mL}$ ), whereas PGT121/PGT122 potency was abrogated upon removal of the $\mathrm{N} 301_{\mathrm{gp} 120}$ glycan (Table 2). Furthermore, PGT122 showed sensitivity to D325 $\mathrm{N}_{\mathrm{gp} 120}$ mutations in the GDIR peptide motif. Taken together, these differences illustrate the divergence of solutions evolved by bNAbs to target this epitope, and BG18's successful strategies to accommodate Env sequence diversity.

Recent studies showed that priming with designed SOSIP Env trimers that bind weakly to the common inferred germline sequence (iGL) of PGT121 and $10-1074^{50}$ could elicit bNAbs resembling PGT121 in PGT121 iGL Ig knock-in mice ${ }^{49}$. 
However, the iGL of BG18 did not bind detectably to the tightestbinding designed priming immunogen $11 \mathrm{MUT}_{\mathrm{B}}$ (Supplementary Figure 11a), which can be rationalized by one of several differences in BG18 and PGT121/10-1074 recognition of Env. First, mature BG18 CDRL2 residues are involved in GDIR recognition with $\mathrm{S} 53_{\mathrm{LC}}$ and Q54 $4_{\mathrm{LC}}$ engaging D325 $5_{\mathrm{gp} 120}$ of GDIR (Fig. 5b). Comparison of iGL and mature BG18 CDRL2 amino acid sequences showed that four of five residues were mutated $\left({ }^{50}\right.$ YKDSE $^{54}$ vs. ${ }^{50}$ SRSSQ $^{54}$, respectively) (Fig. $\left.5 \mathrm{~d}\right)$. Not only does the germline CDRL2 have increased bulk, but also acidic residues flank S53 ${ }_{\mathrm{LC}}$, which interacts with $\mathrm{D} 325_{\mathrm{gp} 120}$. In contrast, $\mathrm{iGL}$ sequences for CDRL1/3 in PGT121/10-1074 show conservation of serines responsible for interacting with D325 $5_{\text {gp } 120}$ in GDIR and flanking residues (Fig. $5 \mathrm{~d}$ ). Thus, since the $11 \mathrm{MUT}_{\mathrm{B}}$ priming immunogen contains no substitutions in GDIR, BG18 iGL likely shows a reduced ability to interact with this region compared with PGT121/10-1074 iGLs. An additional predicted impediment to iGL BG18 binding to $11 \mathrm{MUT}_{\mathrm{B}}$ is the relative proximity of light chain CDRs to the gp120 V1-loop (Fig. 6). Since the CDRs are the most heavily substituted between iGL and mature sequences, altering the chemical properties of this cleft could negatively affect binding. Moreover, $11 \mathrm{MUT}_{\mathrm{B}}$ harbors seven mutations in the V1-loop necessary for iGL PGT121 binding ${ }^{50}$, which likely clash with iGL BG18 given its V1-loop interactions (Supplementary Figure 11b). Finally, mature BG18 is heavily substituted compared to iGL sequences (35 and 26 heavy chain and light chain amino acid mutations, respectively). Previous structural studies of a PGT121 intermediate bound to BG505 Env showed that orientations are defined early during maturation and that differences in amino acid composition can alter antibody footprints on gp $120^{31}$. Thus, it is possible that the conformation of iGL BG18 is incompatible with $11 \mathrm{MUT}_{\mathrm{B}}$ binding.

Although eliciting BG18-like bNAbs would require a different set of designed immunogens than being used for PGT121/101074 bNAbs $^{49,50}$, they might be easier to elicit because of a shorter CDRH3 than PGT121/10-1074 bNAbs. By elucidating the molecular details of BG18's distinct interaction with the V3/ $\mathrm{N} 332_{\text {gp120 }}$ epitope, the structural information reported here will facilitate future immunogen design efforts.

\section{Methods}

Protein expression and purification. Fabs from BG18 (including a N26Q $\mathrm{Q}_{\mathrm{HC}}$ substitution $^{47}$ ), 35O22, and IOMA IgGs were produced as previously described ${ }^{37}$. Briefly, Fabs were expressed by transiently transfecting HEK293-6E cells (National Research Council of Canada) with vectors encoding the appropriate light chain and C-terminal 6x-His tagged heavy chain genes. Secreted Fabs were purified from cell supernatants using $\mathrm{Ni}^{2+}$-NTA affinity chromatography (GE Healthcare), followed by size exclusion chromatography (SEC) with a Superdex200 16/60 column (GE Healthcare). Purified Fabs were concentrated and maintained at $4{ }^{\circ} \mathrm{C}$ in storage buffer $(20 \mathrm{mM}$ Tris $\mathrm{pH} 8.0,150 \mathrm{mM} \mathrm{NaCl}$, and $0.02 \%$ sodium azide).

Genes encoding BG505 SOSIP.664 gp140 20 or B41 SOSIP.664 gp140 ${ }^{61}$ trimers were stably expressed in CHO Flp-In ${ }^{\mathrm{TM}}$ cells (Invitrogen) as described ${ }^{51}$ using cell lines kindly provided by John Moore (Weill Cornell Medical College). Plasmids encoding the BG505 SOSIP.664 gp140 N392 gene variant (see Supplementary Table 1 for primer sequences) was transiently expressed in HEK293-6E cells (National Research Council of Canada) as previously described ${ }^{22}$. In both cases, secreted SOSIP trimers expressed in the absence of kifunensine were isolated from cellular supernatants using 2G12 immunoaffinity chromatography by covalently coupling 2G12 IgG monomer to an activated-NHS Sepharaose column (GE Healthcare). Trimers were eluted using $3 \mathrm{M} \mathrm{MgCl}_{2}$ and immediately dialyzed into storage buffer before SEC purification with a Superdex200 16/60 column (GE Healthcare) against the same buffer. Peak fractions pertaining to SOSIP trimers were pooled and repurified over the same column and buffer conditions. Twelve $1.0-\mathrm{mL}$ fractions were stored separately at $4{ }^{\circ} \mathrm{C}$.

Crystallization of BG18-Env complexes. Complexes for crystallization were produced by an overnight incubation of SOSIP with BG18 and $35 \mathrm{O} 22$ or IOMA Fabs at a 1:1:1 molar ratio, and subsequently concentrated to $5-8 \mathrm{mg} / \mathrm{mL}$ by centrifugation with a $30-\mathrm{kDa}$ concentrator (Amicon). Initial matrix crystallization trials were performed at room temperature using the sitting drop vapor diffusion method by mixing equal volumes of protein sample and reservoir using a TTP LabTech Mosquito robot and commercially available screens (Hampton Research and Qiagen). Initial hits were optimized and crystals were obtained for BG18BG505-35O22 and BG18-B41-35O22 in 0.1 M Tris pH 8.0, 5\% Tacismate pH 8.0, and $14 \%$ polyethylene glycol (PEG) 3350 at $20^{\circ} \mathrm{C}$. BG18-BG505-IOMA crystals were obtained in $0.1 \mathrm{M}$ citric acid pH $3.7,16 \%$ PEG 3350 at $20^{\circ} \mathrm{C}$. Crystals were cryo-protected stepwise with reservoir and a final $20 \% \mathrm{v} / \mathrm{v}$ glycerol concentration before being cryopreserved in liquid nitrogen.

Structure determination and refinement. Conventional X-ray diffraction data were collected for BG18-Env complexes at the Stanford Synchroton Radiation Lightsource (SSRL) beamline $12-2$ on a Pilatus $6 \mathrm{M}$ pixel detector (Dectris). Data from a single crystal for each complex were indexed and integrated in $\mathrm{XDS}^{62}$, and merged with AIMLESS in the CCP4 software suite ${ }^{63}$. Structures were determined by molecular replacement in PHASER ${ }^{64}$ using a single search with coordinates of an a glycosylated gp120-4 protomer (PDB 5T3Z (https://doi.org/10.2210/pdb5T3Z/ pdb)), BG18 Fab (PDB 5UD9 (https://doi.org/10.2210/pdb5UD9/pdb)), and $35 \mathrm{O} 22$ Fab (PDB 4TOY (https://doi.org/10.2210/pdb4TOY/pdb)) or IOMA Fab (PDB 5T3Z (https://doi.org/10.2210/pdb5T3Z/pdb)). Models were refined using B-factor refinement in $\mathrm{CNS}^{65}$ and Phenix ${ }^{56}$, followed by several cycles of manual building with B factor sharpening in $\operatorname{Coot}^{55,66}$. Glycans were initially interpreted and modeled using $F_{\mathrm{o}}-F_{\mathrm{c}}$ maps calculated with model phases contoured at $2 \sigma$, followed by $2 F_{\mathrm{o}}-F_{\mathrm{c}}$ simulated annealing composite omit maps in which modeled glycans were omitted to remove model bias ${ }^{56}$. $N$-linked glycans identified at individual PNGSs in our crystallographic studies on both BG505.664 and B41.664 SOSIP trimers were generally consistent with the mixture of glycans observed by mass spectroscopy ${ }^{18,57}$ and previous crystallographic studies of a natively and fully glycosylated Env trimer ${ }^{25}$. Therefore, modeling of complex-type glycans at positions N88gp120 and N156gp120 were primarily determined by the presence of electron density characteristic of a core fucose, which when modeled, slightly lowered $R_{\text {free }}$ values. Additional details of glycan modeling and coordinate refinement were followed as previously described ${ }^{25,67}$. Inclusion of higher resolution data with weak intensities improved refinement behavior and stereochemistry as described ${ }^{68}$.

XFEL diffraction experiments were performed at the MFX endstation of LCLS using a standard goniometer setup ${ }^{48}, 9.5 \mathrm{keV}$ X-ray pulses with $40 \mathrm{fs}$ duration and a $5-\mu \mathrm{m}$ beam focus at the crystal interaction point. Diffraction images were recorded on a Rayonix MX325 detector data and were integrated with IOTA ${ }^{69}$ using the data reduction algorithms implemented in cctbx.xfel ${ }^{70}$. Of the 627 collected images, 589 contained discernible diffraction; of these, 570 were successfully integrated and yielded correct crystal symmetry and unit cell values. Scaling, post-refinement, and merging was carried out using PRIME ${ }^{71}$. Of the 570 integrated diffraction images, 526 were included in the final merged dataset, which was complete (99.1\%) to $3.8 \AA$, and exhibited good multiplicity (9.2-fold) and reasonable merging statistics (Table 1). Phases were generated by molecular replacement, using our refined $4.1 \AA$ synchrotron structure with glycans omitted as a search model.

Superposition and figures were rendered using PyMOL (Version 1.5.0.4 Schrodinger, LLC), and protein electrostatic calculations were achieved using APBS and PDB2PQR webservers ${ }^{72}$. BSAs were determined with PDBePISA using a $1.4-\AA$ probe $^{73}$. Potential hydrogen bonds were assigned using a distance of $<3.6 \AA$ and an A-D-H angle of $>90^{\circ}$, while the maximum distance allowed for a van der Waals interaction was $4.0 \AA$ A Putative $\mathrm{H}$-bonds, van der Waals assignments, and total BSA should be considered tentative, owing to the relatively low structure resolutions.

Binding experiments. SPR experiments were carried out on a Biacore T100 (Biacore) using a standard single-cycle kinetics method as previously described $^{22,37}$. Briefly, a CM5 chip, primarily amine coupled with Protein A, was used to immobilize 8ANC195 IgG, a gp120-gp41 interface bNAb ${ }^{37}$. Remaining Protein A sites were blocked with $1 \mu \mathrm{M}$ human Fc. BG505 HIV-1 SOSIP trimers were captured by injecting $10 \mu \mathrm{M}$ solutions at a flow rate of $30 \mu \mathrm{L} / \mathrm{s}$ for $180 \mathrm{~s}$. Mature BG18 Fab was injected at decreasing concentrations (3-fold dilution series with a starting top concentration of $110 \mathrm{nM}$ ) at $30 \mu \mathrm{L} / \mathrm{s}$ for $60 \mathrm{~s}$ and allowed to dissociated for 300 $\mathrm{s}$. Kinetic analyses were done after subtraction of reference curves to obtain $k_{\mathrm{a}}, k_{\mathrm{d}}$, and $K_{\mathrm{D}}$ values for a 1:1 binding model with or without a bulk refractive index change correction as appropriate (Biacore T200 Evaluation software).

An ELISA to evaluate binding of mature and iGL BG18 IgG to $11 \mathrm{MUT}_{\mathrm{B}} \operatorname{SOSIP}^{50}$ was performed by coating of High-Bind 96-well plates (Corning \#9018) with $50 \mu \mathrm{L}$ per well of a $2-\mu \mathrm{g} / \mathrm{mL}$ solution of purified $11 \mathrm{MUT}_{\mathrm{B}}$ in PBS overnight at $4{ }^{\circ} \mathrm{C}$. Plates were washed six times with washing buffer ( $1 \times$ PBS with $0.05 \%$ Tween 20 (SigmaAldrich) ) and incubated in blocking buffer (1× PBS with $1 \%$ non-fat milk) for $1 \mathrm{~h}$ at room temperature (RT). Immediately after blocking, IgGs were added in blocking buffer and incubated for $2 \mathrm{~h}$ at RT. Antibodies were assayed at a $5-\mu \mathrm{g} / \mathrm{mL}$ starting dilution and seven additional 3 -fold serial dilutions. Plates were washed six times with washing buffer and then incubated with anti-human IgG secondary antibody conjugated to horseradish peroxidase (HRP) (Jackson Laboratories) in washing buffer at a 1:5000 dilution. Plates were developed by addition of the HRP substrate, ABTS (Life Technologies), and absorbance was measured at $405 \mathrm{~nm}$ with an ELISA microplate reader (FluoStar Omega, BMG Labtech). 
Data availability. Coordinates and structure factors reported in this manuscript have been deposited in the Protein Data Bank with accession codes $6 \mathrm{CH} 7,6 \mathrm{CH} 8$, $6 \mathrm{CH} 9$, and $6 \mathrm{CHB}$. Other data are available from the corresponding author upon reasonable request.

Received: 1 December 2017 Accepted: 1 March 2018

Published online: 28 March 2018

\section{References}

1. Klein, F. et al. HIV therapy by a combination of broadly neutralizing antibodies in humanized mice. Nature 492, 118-122 (2012).

2. Horwitz, J. A. et al. HIV-1 suppression and durable control by combining single broadly neutralizing antibodies and antiretroviral drugs in humanized mice. Proc. Natl Acad. Sci. USA 110, 16538-16543 (2013).

3. Shingai, M. et al. Antibody-mediated immunotherapy of macaques chronically infected with SHIV suppresses viraemia. Nature 503, 277-280 (2013).

4. Barouch, D. H. et al. Therapeutic efficacy of potent neutralizing HIV-1specific monoclonal antibodies in SHIV-infected rhesus monkeys. Nature 503 , 224-228 (2013).

5. Bar, K. J. et al. Effect of HIV Antibody VRC01 on Viral Rebound after Treatment Interruption. N. Engl. J. Med. 375, 2037-2050 (2016).

6. Schoofs, T. et al. HIV-1 therapy with monoclonal antibody 3BNC117 elicits host immune responses against HIV-1. Science 352, 997-1001 (2016).

7. Caskey, M. et al. Viraemia suppressed in HIV-1-infected humans by broadly neutralizing antibody 3BNC117. Nature 522, 487-491 (2015).

8. Caskey, M. et al. Antibody 10-1074 suppresses viremia in HIV-1-infected individuals. Nat. Med. 23, 185-191 (2017).

9. Lynch, R. M. et al. Virologic effects of broadly neutralizing antibody VRC01 administration during chronic HIV-1 infection. Sci. Transl. Med. 7 319ra206-319ra206 (2015).

10. Klein, F. et al. Somatic mutations of the immunoglobulin framework are generally required for broad and potent HIV-1 neutralization. Cell 153 126-138 (2013).

11. West, A. P. et al. Structural insights on the role of antibodies in HIV-1 vaccine and therapy. Cell 156, 633-648 (2014).

12. West, A. P. J., Diskin, R., Nussenzweig, M. C. \& Bjorkman, P. J. Structural basis for germ-line gene usage of a potent class of antibodies targeting the CD4-binding site of HIV-1gp120. Proc. Natl Acad. Sci. USA 109, E2083-E2090 (2012)

13. Dingens, A. S., Haddox, H. K., Overbaugh, J. \& Bloom, J. D. Comprehensive mapping of HIV-1 escape from a broadly neutralizing antibody. Cell Host Microbe 21, 777-787.e4 (2017).

14. Klein, J. S. \& Bjorkman, P. J. Few and far between: how HIV may be evading antibody avidity. PLoS Pathog. 6, el000908 (2010).

15. Galimidi, R. P. et al. Intra-spike crosslinking overcomes antibody evasion by HIV-1. Cell 160, 433-446 (2015).

16. Wei, X. et al. Antibody neutralization and escape by HIV-1. Nature $\mathbf{4 2 2}$, 307-312 (2003).

17. Zhu, X., Borchers, C., Bienstock, R. J. \& Tomer, K. B. Mass spectrometric characterization of the glycosylation pattern of HIV-gp120 expressed in CHOcells. Biochemistry 39, 11194-11204 (2000).

18. Behrens, A.-J. et al. Composition and antigenic effects of individual glycan sites of a trimeric HIV-1 envelope glycoprotein. Cell Rep. 14, 2695-2706 (2016).

19. Binley, J. M. et al. Role of complex carbohydrates in human immunodeficiency virus type 1 infection and resistance to antibody neutralization. J. Virol. 84, 5637-5655 (2010).

20. Sanders, R. W. et al. A next-generation cleaved, soluble HIV-1 Env trimer, BG505 SOSIP.664gp140, expresses multiple epitopes for broadly neutralizing but not non-neutralizing antibodies. PLoS Pathog. 9, el003618 (2013).

21. Ward, A. B. \& Wilson, I. A. The HIV-1 envelope glycoprotein structure: nailing down a moving target. Immunol. Rev. 275, 21-32 (2017).

22. Scharf, L. et al. Broadly neutralizing antibody $8 \mathrm{ANC} 195$ recognizes closed and open states of HIV-1 env. Cell 162, 1379-1390 (2015).

23. Sievers, S. A., Scharf, L., West, A. P. Jr. \& Bjorkman, P. J. Antibody engineering for increased potency, breadth and half-life. Curr. Opin. HIV AIDS 10, 151-159 (2015).

24. Diskin, R. et al. Increasing the potency and breadth of an HIV antibody by using structure-based rational design. Science 334, 1289-1293 (2011).

25. Gristick, H. B. et al. Natively glycosylated HIV-1 Env structure reveals new mode for antibody recognition of the CD4-binding site. Nat. Struct. Mol. Biol. 23, 906-915 (2016).

26. Pancera, M. et al. Crystal structures of trimeric HIV envelope with entry inhibitors BMS-378806 and BMS-626529. Nat. Chem. Biol. 68, 4031-1122 (2017).
27. Stewart-Jones, G. B. E. et al. Trimeric HIV-1-env structures define glycan shields from clades A, B, and G. Cell 165, 813-826 (2016)

28. Kwon, Y. D. et al. Crystal structure, conformational fixation and entry-related interactions of mature ligand-free HIV-1 Env. Nat. Struct. 22, 522-531 (2015)

29. Pancera, M. et al. Structure and immune recognition of trimeric pre-fusion HIV-1 Env. Nature 514, 455-461 (2014).

30. Kwong, P. D. et al. Structure of an HIV gp120 envelope glycoprotein in complex with the CD4 receptor and a neutralizing human antibody. Nature 393, 648-659 (1998).

31. Garces, F. et al. Affinity maturation of a potent family of HIV antibodies is primarily focused on accommodating or avoiding glycans. Immunity $\mathbf{4 3}$, 1053-1063 (2015).

32. Garces, F. et al. Structural evolution of glycan recognition by a family of potent HIV antibodies. Cell 159, 69-79 (2014).

33. Julien, J.-P. et al. Crystal structure of a soluble cleaved HIV-1 envelope trimer Science 342, 1477-1483 (2013).

34. Diskin, R., Marcovecchio, P. M. \& Bjorkman, P. J. Structure of a clade C HIV-1 gp120 bound to CD4 and CD4-induced antibody reveals anti-CD4 polyreactivity. Nat. Struct. 17, 1-19 (2010).

35. Kong, R., Xu, K., Zhou, T., Acharya, P. \& Lemmin, T. Fusion peptide of HIV-1 a site of vulnerability to neutralizing antibody. Science 352, 828-833 (2016).

36. Zhou, T. et al. Structural basis for broad and potent neutralization of HIV-1 by antibody VRC01. Science 329, 811-817 (2010).

37. Scharf, L. et al. Antibody 8 ANC195 reveals a site of broad vulnerability on the HIV-1 envelope spike. Cell Rep. 7, 785-795 (2014).

38. Lee, J. H., Ozorowski, G. \& Ward, A. B. Cryo-EM structure of a native, fully glycosylated, cleaved HIV-1 envelope trimer. Science 351, 1043-1048 (2016).

39. Lee, J. H., de Val, N., Lyumkis, D. \& Ward, A. B. Model building and refinement of a natively glycosylated HIV-1 env protein by high-resolution cryoelectron microscopy. Structure 23, 1943-1951 (2015).

40. Mouquet, $\mathrm{H}$. et al. Complex-type N-glycan recognition by potent broadly neutralizing HIV antibodies. Proc. Natl Acad. Sci. USA 109, E3268-E3277 (2012).

41. Walker, L. M. et al. Broad neutralization coverage of HIV by multiple highly potent antibodies. Nature 477, 466-470 (2011).

42. Sok, D. et al. A prominent site of antibody vulnerability on HIV envelope incorporates a motif associated with CCR5 binding and its camouflaging glycans. Immunity 45, 31-45 (2016).

43. Doores, K. J. et al. Two classes of broadly neutralizing antibodies within a single lineage directed to the high-mannose patch of HIV envelope. J. Virol. 89, 1105-1118 (2015)

44. Kong, L. et al. Supersite of immune vulnerability on the glycosylated face of HIV-1 envelope glycoprotein gp120-803. Nat. Struct. Mol. Biol. 20, 796-803 (2013).

45. Pejchal, R. et al. A potent and broad neutralizing antibody recognizes and penetrates the HIV glycan shield. Science 334, 1097-1103 (2011).

46. Andrabi, R. et al. Glycans function as anchors for antibodies and help drive HIV broadly neutralizing antibody development. Immunity 47, 524-537.e3 (2017).

47. Freund, N. T. et al. Coexistence of potent HIV-1 broadly neutralizing antibodies and antibody-sensitive viruses in a viremic controller. Sci. Transl. Med. 9, eaal2144 (2017).

48. Cohen, A. E. et al. Goniometer-based femtosecond crystallography with X-ray free electron lasers. Proc. Natl Acad. Sci. USA 111, 17122-17127 (2014).

49. Escolano, A. et al. Sequential immunization elicits broadly neutralizing antiHIV-1 antibodies in Ig knockin mice. Cell 166, 1445-1458.e12 (2016).

50. Steichen, J. M. et al. HIV vaccine design to target germline precursors of glycan-dependent broadly neutralizing antibodies. Immunity 45, 483-496 (2016)

51. Chung, N. P. et al. Stable $293 \mathrm{~T}$ and $\mathrm{CHO}$ cell lines expressing cleaved, stable HIV-1 envelope glycoprotein trimers for structural and vaccine studies. Retrovirology 11, 33 (2014).

52. Go, E. P. et al. Glycosylation benchmark profile for HIV-1 envelope glycoprotein production based on eleven env trimers. J. Virol. 91, e02428-16-19 (2017)

53. Huang, J. et al. Broad and potent HIV-1 neutralization by a human antibody that binds the gp41-120 interface. Nature 515, 138-142 (2014).

54. Zhou, Q. et al. Architecture of the synaptotagmin-SNARE machinery for neuronal exocytosis. Nature 525, 62-67 (2015).

55. Liu, C. \& Xiong, Y. Electron density sharpening as a general technique in crystallographic studies. J. Mol. Biol. 426, 980-993 (2014).

56. Adams, P. D. et al. PHENIX: a comprehensive Python-based system for macromolecular structure solution. Acta Crystallogr. D Biol. Crystallogr. 66, 213-221 (2010).

57. Cao, L. et al. Global site-specific N-glycosylation analysis of HIV envelope glycoprotein. Nat. Commun. 8, 1-13 (2017). 
58. Zhou, T. et al. Quantification of the impact of the HIV-1-glycan shield on antibody elicitation. Cell Rep. 19, 719-732 (2017).

59. Wu, X. et al. Focused evolution of HIV-1 neutralizing antibodies revealed by structures and deep sequencing. Science 333, 1593-1602 (2011).

60. Zhou, T. et al. Multidonor analysis reveals structural elements, genetic determinants, and maturation pathway for HIV-1 neutralization by VRC01class antibodies. Immunity 39, 245-258 (2013).

61. Pugach, P. et al. A native-like SOSIP.664 trimer based on an HIV-1 subtype B env gene. J. Virol. 89, 3380-3395 (2015).

62. Kabsch, W. XDS. Acta Crystallogr. D Biol. Crystallogr. 66, 125-132 (2010).

63. Winn, M. D. et al. Overview of the CCP4 suite and current developments. Acta Crystallogr. D Biol. Crystallogr. 67, 235-242 (2011).

64. McCoy, A. J. et al. Phaser crystallographic software. J. Appl. Crystallogr. 40, 658-674 (2007).

65. Brunger, A. T. et al. Crystallography \& NMR system: a new software suite for macromolecular structure determination. Acta Crystallogr. D Biol. Crystallogr. 54, 905-921 (1998).

66. Emsley, P., Lohkamp, B., Scott, W. G. \& Cowtan, K. Features and development of Coot. Acta Crystallogr. D Biol. Crystallogr. 66, 486-501 (2010).

67. Gristick, H. B., Wang, H. \& Bjorkman, P. J. X-ray and EM structures of a natively glycosylated HIV-1 envelope trimer. Acta Crystallogr. D Struct. Biol. 73, 822-828 (2017).

68. Karplus, P. A. \& Diederichs, K. Linking crystallographic model and data quality. Science 336, 1030-1033 (2012).

69. Lyubimov, A. Y. et al. IOTA: integration optimization, triage and analysis tool for the processing of XFEL diffraction images. J. Appl. Crystallogr. 49, 1057-1064 (2016).

70. Hattne, J. et al. Accurate macromolecular structures using minimal measurements from X-ray free-electron lasers. Nat. Methods 11, 545-548 (2014).

71. Uervirojnangkoorn, M. et al. Enabling X-ray free electron laser crystallography for challenging biological systems from a limited number of crystals. Elife 4, 213 (2015).

72. Unni, S. et al. Web servers and services for electrostatics calculations with APBS and PDB2PQR. J. Comput. Chem. 32, 1488-1491 (2011).

73. Krissinel, E. \& Henrick, K. Inference of macromolecular assemblies from crystalline state. J. Mol. Biol. 372, 774-797 (2007).

74. West, A. P. et al. Computational analysis of anti-HIV-1 antibody neutralization panel data to identify potential functional epitope residues. Proc. Natl Acad. Sci. USA 110, 10598-10603 (2013).

\section{Acknowledgements}

We thank J. Vielmetter, S. Khomandiak, and the Caltech Protein Expression Center for producing proteins, J. Kaiser and the beamline staff at SSRL for data collection assistance, and members of the Bjorkman and Nussenzweig laboratories for critical reading of the manuscript. We also thank Silvia Russi, Clyde Smith, Jinhu Song, and Alexander Batyuk for user support during our MFX experiment and the lab of Guillermo Calero (University of Pittsburgh) for providing loop supports to mount multiple crystals during XFEL experiments. C.O.B holds a Hanna Gray Fellowship from the Howard Hughes Medical Institute and a Postdoctoral Enrichment Program Award from the Burroughs Wellcome Fund. This research was supported by the National Institute Of Allergy And Infectious Diseases of the National Institutes of Health Grant HIVRAD P01 AI100148 (P.J.B.); (the content is solely the responsibility of the authors and does not necessarily represent the official views of the National Institutes of Health), the Bill and Melinda Gates Foundation (Collaboration for AIDS Vaccine Discovery Grant OPP1124068 (M.C.N./P.J B.)), and the Molecular Observatory at Caltech supported by the Gordon and Betty Moore Foundation. Use of the Linac Coherent Light Source (LCLS) and use of the Stanford Synchrotron Radiation Lightsource (SSRL), SLAC National Accelerator Laboratory, is supported by the U.S. Department of Energy, Office of Science, Office of Basic Energy Sciences under Contract No. DE-AC02-76SF00515. The SSRL Structural Molecular Biology Program is supported by the US Department of Energy Office of Biological and Environmental Research, and by the National Institutes of Health (NIH), National Institute of General Medical Sciences (including P41GM103393).

\section{Author contributions}

C.O.B., A.P.W. Jr., M.C.N., and P.J.B. conceived the experiments; C.O.B. optimized the crystallography conditions, solved and analyzed the crystal structures; C.O.B., H.B.G., and A.P.W. Jr. performed computational and structural analyses of BG18-class and PGT121/10-1074-class antibodies; C.O.B. and H.B.G. purified the proteins, performed and analyzed SPR binding experiments; C.O.B., A.Y.L., and A.E.C. performed the XFEL data collection and analyses; N.T.F. was responsible for isolating BG18 monoclonal antibodies; A.E. and H.H. performed ELISA experiments; C.O.B., A.P.W., M.C.N., and P. J.B. wrote the paper, on which all principal investigators and authors commented.

\section{Additional information}

Supplementary Information accompanies this paper at https://doi.org/10.1038/s41467018-03632-y.

Competing interests: The authors declare no competing interests.

Reprints and permission information is available online at http://npg.nature.com/ reprintsandpermissions/

Publisher's note: Springer Nature remains neutral with regard to jurisdictional claims in published maps and institutional affiliations.

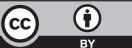

Open Access This article is licensed under a Creative Commons Attribution 4.0 International License, which permits use, sharing, adaptation, distribution and reproduction in any medium or format, as long as you give appropriate credit to the original author(s) and the source, provide a link to the Creative Commons license, and indicate if changes were made. The images or other third party material in this article are included in the article's Creative Commons license, unless indicated otherwise in a credit line to the material. If material is not included in the article's Creative Commons license and your intended use is not permitted by statutory regulation or exceeds the permitted use, you will need to obtain permission directly from the copyright holder. To view a copy of this license, visit http://creativecommons.org/ licenses/by/4.0/.

(C) The Author(s) 2018 